\title{
Waveguide integrated graphene mid-infrared photodetector
}

\author{
Z.Qu*a, M. Nedeljkovic ${ }^{\mathrm{a}}$, Y. Wu ${ }^{\mathrm{a}}$, J. Soler Penades ${ }^{\mathrm{a}}$, A. Z. Khokhar ${ }^{\mathrm{a}}$, W. Cao ${ }^{\mathrm{a}}$, A. M. Osman ${ }^{\mathrm{a}}$, \\ Y. Qi ${ }^{\mathrm{a}}$, N. K. Aspiotis ${ }^{\mathrm{a}}$, K. A. Morgan ${ }^{\mathrm{a}}$, C. C. Huang ${ }^{\mathrm{a}}$, G. Z. Mashanovich ${ }^{\mathrm{a}}$ \\ ${ }^{a}$ Optoelectronics Research Centre, University of Southampton, Southampton, Hampshire, SO17 1BJ, \\ United Kingdom
}

\begin{abstract}
Group IV platforms can operate at longer wavelengths due to their low material losses. By combining graphene and $\mathrm{Si}$ and Ge platforms, photodetection can be achieved by using graphene's optical properties and coplanar integration methods. Here, we presented a waveguide coupled graphene photodetector operating at a wavelength of $3.8 \mu \mathrm{m}$.
\end{abstract}

Keywords: Silicon photonics, mid-infrared, graphene photodetector

\section{INTRODUCTION}

Group IV material photonic integrated circuit platforms have the ability to benefit from graphene's superb electronic and optical properties, in order to address applications in areas such as environmental and bio-chemical sensing, homeland security, medicine or astronomy. Silicon-on-insulator waveguides, which are commonly used in the near-infrared, also have low propagation loss in the 3-4 $\mu \mathrm{m}$ wavelength range. Here, we present the first graphene photodetector based on the coplanar integration method with silicon-on-insulator waveguide and transferred CVD graphene that operates at 3.8 $\mu \mathrm{m}$ wavelength.

\section{MID-INFRARED GRAPHENE PHOTODETECTORS}

Mid-infrared (MIR) silicon photonics can benefit from the maturity of CMOS processing and from demonstrated devices in the telecom wavelength range as many of the techniques can be used at longer wavelengths [1]. The silicon-oninsulator (SOI) platform has been widely implemented in MIR silicon photonics. SOI waveguides are low loss up to 4 $\mu \mathrm{m}$ and waveguide propagation loss as low as $1.3 \pm 0.6 \mathrm{~dB} / \mathrm{cm}$ has been demonstrated at a wavelength of $\sim 3.8 \mu \mathrm{m}$ [2].

2D materials have several advantages compared to conventional 3D materials, and in particular better quantum confinement in the direction perpendicular to the 2D plane, that leads to distinctive optical and electronic properties. 2D materials can be integrated with other materials using waveguides and cavities to form heterostructures [3].

Due to graphene's gapless band structure it acts as semimetal and can interact with light from microwave to ultraviolet wavelengths. The absorbed photons can be converted to electron hole pairs and move to electrodes quickly due to graphene's fast carrier mobility, which makes it a promising material for light detection or manipulation [4]. Graphene has excellent properties [5] such as high carrier mobility, thermal conductivity, and linear dispersion [6] as well as high optical absorption across a wide range of wavelengths. The optical absorption mechanisms in graphene are divided into two processes: interband transitions and intraband transitions. Interband transitions occur when one electron in the "valance" band of graphene absorbs a photon and is excited to an empty state in the "conduction" band with the same momentum [7]. The interband transition is only permitted under the condition that the energy of filled state is larger than the absolute value of the chemical potential. Otherwise, interband transitions will be prevented by Pauli blocking. On the other hand, intraband transition occurs under a phonon assisted process, which happens in the far-infrared (FIR) to terahertz wavelength regions. Interband transitions can be suppressed if the chemical potential is tuned close to the Dirac energy. With the control of the absorption mechanism, waveguide-coupled graphene optoelectronic devices have potential to operate in the MIR wavelength region, and under such circumstances the absorption mechanism is dominated by the interband transition [8-10]. Thus, the combination of low loss silicon waveguides and high absorption nature of graphene is promising for light detection in the MIR. 
Single layer graphene was first demonstrated by Novoselov, Geim and their colleagues by graphite exfoliation in 2004 [11]. By using the coplanar integration method, the optical mode propagates in the silicon waveguides and interacts with graphene at the same time, thus achieving a greater interaction length compared to the normal incidence situation in which the interaction length is limited by the thickness of graphene. In terms of photodetection, by using the coplanar integration configuration, the light-graphene interaction is determined by the length of the device, rather than the thickness of the graphene as in the normal incidence situation. Thus, coplanar integration is a promising solution for high efficiency graphene integrated photodetectors [6, 12-15]. The first demonstration of a graphene photodetector was based on a graphene transistor, in which the metal-graphene junction was illuminated by infrared and visible light, achieving $40 \mathrm{GHz}$ bandwidth at room temperature [16]. However, responsivities were limited to few $\mathrm{mA} / \mathrm{W}$ at telecom wavelengths. After optimizing the device configuration, photoresponse of graphene photodetectors has been increased to few A/W by several approaches, such as integration of graphene transistor and optical cavities [17], light interaction enhanced by introducing plasmonic antennas $[18,19]$, or by using in-plane evanescent filed enhanced light/graphene interaction [12-14, 20, 21].

\section{DESIGN OF THE WAVEGUIDE INTEGRATED GRAPHENE PHOTODETECTOR}

Here we present a waveguide integrated metal-graphene-metal (M-G-M) photodetector with $500 \mu \mathrm{m}$ long graphene section and asymmetric electrodes separation to the waveguide at $3.8 \mu \mathrm{m}$ wavelength operating at room temperature. The schematic of the cross-section of the device is shown in Figure 1. The first step of fabrication was to use a SOI wafer to form rib waveguides, with a width of $1300 \mathrm{~nm}$, height of $500 \mathrm{~nm}$, and slab region thickness of $50 \mathrm{~nm}$. A $90 \mathrm{~nm}$ thick PECVD $\mathrm{SiO}_{2}$ layer was then deposited for passivation and reducing charging effects. The next step was to transfer the graphene onto the chip surface, and to pattern it by reactive ion etching (RIE). Finally, $100 \mathrm{~nm}$ thick Au electrodes were fabricated by using the lift-off patterning method. Au contacts were positioned on top of the graphene on either side of the waveguide core, with a separation of $1.5 \mu \mathrm{m}$ on one side and $5 \mu \mathrm{m}$ on the other.

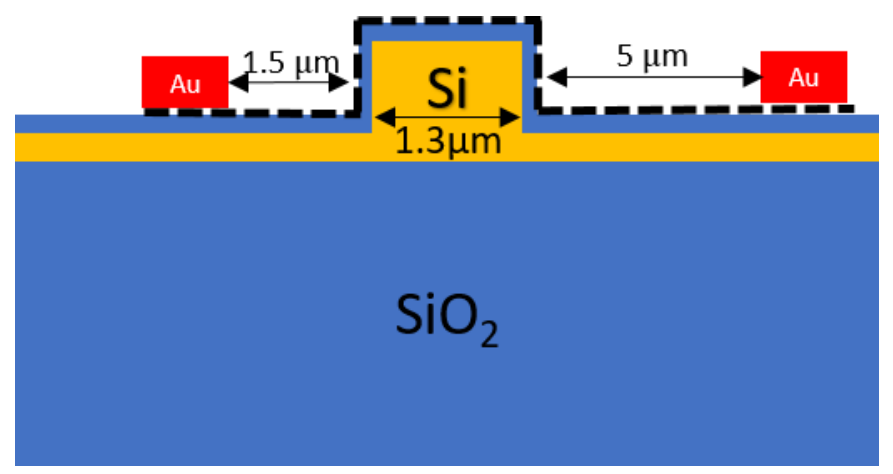

Figure 1. Schematic cross-section diagram of the device.

\section{CHARACTERISATION}

A tunable quantum cascade laser (QCL) operating at 3.7-3.9 $\mu \mathrm{m}$ under continuous wave (CW) mode with transverse electric (TE) polarisation was used as a light source. The light was coupled into the Si waveguides via grating couplers using fluoride based MIR fibres. By using the cut-back method, the normalised transmission of the waveguides covered with graphene was measured, and is plotted in Figure 2. The gradient of the linear fitting indicates that absorption due to graphene on the waveguide surface was $150 \mathrm{~dB} / \mathrm{cm}$ at the $3.8 \mu \mathrm{m}$ wavelength.

In terms of the optoelectronic characterisation, the device was characterised under $\pm 1 \mathrm{~V}$ bias voltage. Bias voltage was applied to the two electrodes. Generated photocurrent was measured by a picoammeter (Keithley 6487). Figure 3 plots the photocurrent as a function of the increased optical power that was coupled into the graphene photodetector. The gradient of the linear fitting gives the photoresponsivity of the device at $3.8 \mu \mathrm{m}$ under $-1 \mathrm{~V}$ bias voltage of $2.2 \mathrm{~mA} / \mathrm{W}$. The optical power incident on the photodetector was calculated by taking into account the absorption from the input fibre, the coupling loss of the input grating coupler, and the loss from access waveguides. 


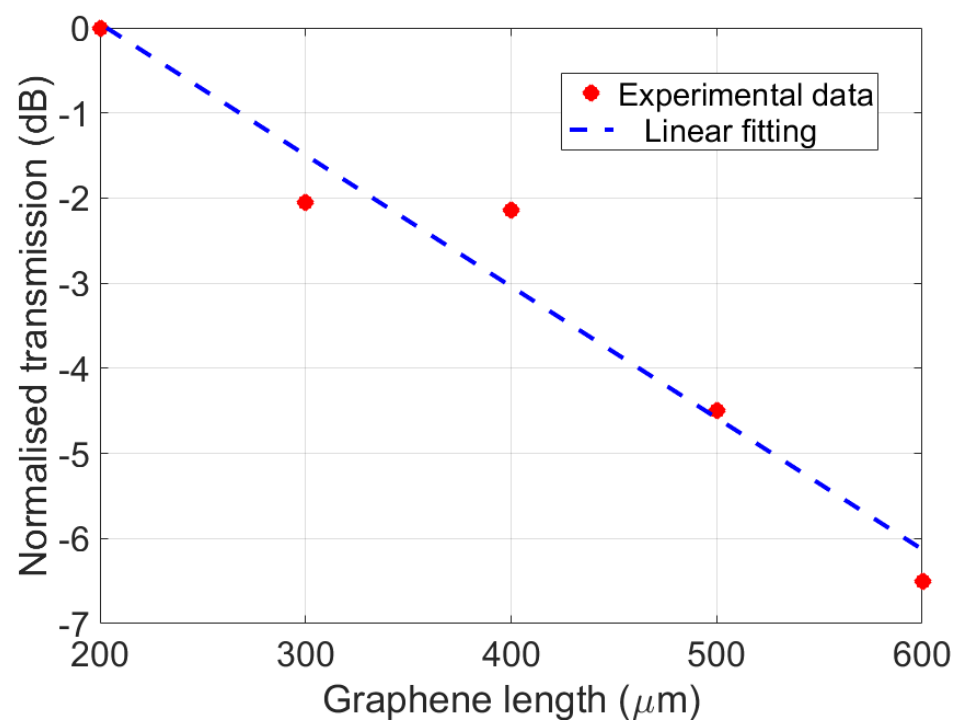

Figure 2. The normalized transmission of waveguides covered by increased length of graphene. The gradient of the linear fitting is the graphene absorption coefficient, which is $150 \mathrm{~dB} / \mathrm{cm}$ at $3.8 \mu \mathrm{m}$ wavelength.

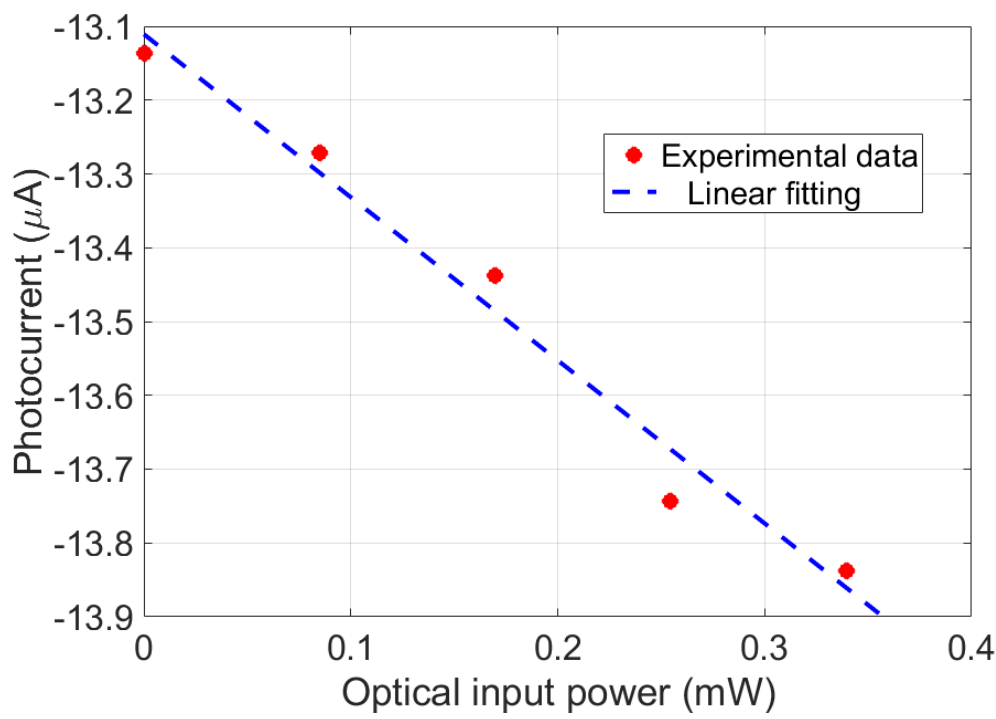

Figure 3. Photocurrent versus optical input power coupled into the detector under $-1 \mathrm{~V}$ bias voltage. The gradient of the linear fitting curve represents the photoresponsivity of the device, which is $2.2 \mathrm{~mA} / \mathrm{W}$ at $3.8 \mu \mathrm{m}$ wavelength.

Figure 4 shows the calculated photoresponsivities as a function of the bias voltage. From the graph, it can be seen that the negative voltage side shows the linear relation between the bias voltage and the photoresponsivity.

Figure 5 shows the spectrum of the transferred graphene on waveguides with $90 \mathrm{~nm} \mathrm{SiO}_{2}$ after all the fabrication processes. $\mathrm{G}$ and $2 \mathrm{D}$ peaks can be seen in the spectrum but also the defects peaks, which indicates that the single crystal lattice was damaged during the fabrication. The maximum photoresponsivity of $2.2 \mathrm{~mA} / \mathrm{w}$ has been measured under $-1 \mathrm{~V}$ bias voltage at $3.8 \mu \mathrm{m}$ wavelength There are some factors that can be optimised to improve the photoresponsivity of the device, such as optimising waveguide geometry to increase the overlap between the optical mode and graphene, adjusting the separations between the Au electrodes and the Si waveguide, modifying fabrication processes to have better graphene quality. Therefore, it will be possible to obtain higher responsivity of the waveguide integrated graphene detectors. 


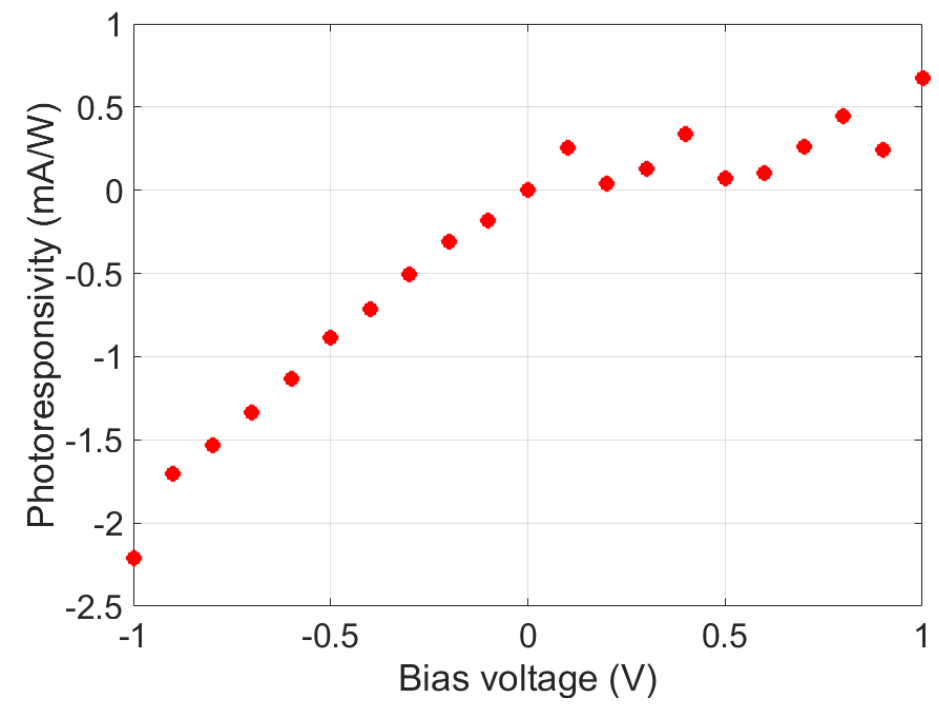

Figure 4. Photoresponsivity versus bias voltage of the graphene photodetector at $3.8 \mu \mathrm{m}$ wavelength. The highest responsivity appeared at $-1 \mathrm{~V}$.

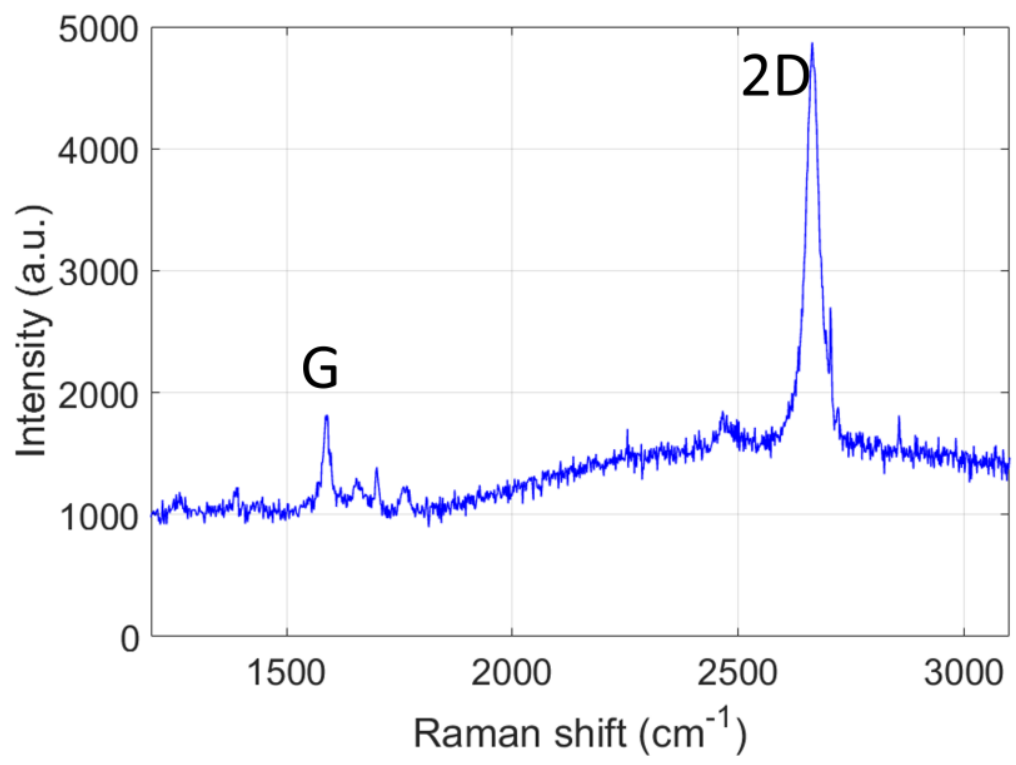

Figure 5. Raman spectrum of transferred graphene on waveguides after metallization. G and 2D peaks show the existence of single layer graphene and defects peaks indicate defects in the graphene structure.

\section{CONCLUSION}

In summary, we have demonstrated the first waveguide integrated graphene photodetector operating at $3.8 \mu \mathrm{m}$ wavelength. Maximum photoresponsivity of $2.2 \mathrm{~mA} / \mathrm{W}$ has been observed under $-1 \mathrm{~V}$ bias voltage. The responsivity can be improved by improved design and fabrication of the detector. There is high potential for the implementation of waveguide integrated graphene photodetectors in the mid-infrared for various applications. 


\section{REFERENCES}

[1] R. Soref, "Mid-infrared photonics in silicon and germanium," Nature photonics, 4(8), 495-497 (2010).

[2] G. Z. Mashanovich, F. Y. Gardes, D. J. Thomson et al., "Silicon photonic waveguides and devices for near-and mid-IR applications," IEEE Journal of Selected Topics in Quantum Electronics, 21(4), 407-418 (2015).

[3] K. F. Mak, L. Ju, F. Wang et al., "Optical spectroscopy of graphene: from the far infrared to the ultraviolet," Solid State Communications, 152(15), 1341-1349 (2012).

[4] F. Koppens, T. Mueller, P. Avouris et al., "Photodetectors based on graphene, other two-dimensional materials and hybrid systems," Nature nanotechnology, 9(10), 780-793 (2014).

[5] F. Xia, H. Wang, D. Xiao et al., "Two-dimensional material nanophotonics," Nature Photonics, 8(12), 899-907 (2014).

[6] L. Vicarelli, M. Vitiello, D. Coquillat et al., "Graphene field-effect transistors as room-temperature terahertz detectors," Nature materials, 11(10), 865-871 (2012).

[7] A. Kuzmenko, E. Van Heumen, F. Carbone et al., "Universal optical conductance of graphite," Physical review letters, 100(11), 117401 (2008).

[8] Z. Li, E. A. Henriksen, Z. Jiang et al., "Dirac charge dynamics in graphene by infrared spectroscopy,” Nature Physics, 4(7), 532-535 (2008).

[9] P. Avouris, and M. Freitag, "Graphene photonics, plasmonics, and optoelectronics," IEEE Journal of selected topics in quantum electronics, 20(1), 72-83 (2014).

[10] P. Avouris, "Graphene: electronic and photonic properties and devices," Nano letters, 10(11), 4285-4294 (2010).

[11] K. S. Novoselov, A. K. Geim, S. V. Morozov et al., "Electric Field Effect in Atomically Thin Carbon Films," Science, 306(5696), 666-669 (2004).

[12] X. Gan, R.-J. Shiue, Y. Gao et al., "Chip-integrated ultrafast graphene photodetector with high responsivity," Nature Photonics, 7(11), 883-887 (2013).

[13] X. Wang, Z. Cheng, K. Xu et al., "High-responsivity graphene/silicon-heterostructure waveguide photodetectors," Nature Photonics, 7, 888 (2013).

[14] A. Pospischil, M. Humer, M. M. Furchi et al., "CMOS-compatible graphene photodetector covering all optical communication bands," Nature Photonics, 7(11), 892-896 (2013).

[15] H. Li, Y. Anugrah, S. J. Koester et al., "Optical absorption in graphene integrated on silicon waveguides," Applied Physics Letters, 101(11), 111110 (2012).

[16] F. Xia, T. Mueller, Y.-m. Lin et al., "Ultrafast graphene photodetector," Nature nanotechnology, 4(12), 839-843 (2009).

[17] M. Engel, M. Steiner, A. Lombardo et al., "Light-matter interaction in a microcavity-controlled graphene transistor," Nature communications, 3, 906 (2012).

[18] Z. Fang, Z. Liu, Y. Wang et al., “Graphene-antenna sandwich photodetector,” Nano letters, 12(7), 3808-3813 (2012).

[19] T. Echtermeyer, L. Britnell, P. Jasnos et al., "Strong plasmonic enhancement of photovoltage in graphene," arXiv preprint arXiv:1107.4176, (2011).

[20] S. J. Koester, and M. Li, "Waveguide-coupled graphene optoelectronics," IEEE Journal of Selected Topics in Quantum Electronics, 20(1), 84-94 (2014).

[21] I. Goykhman, U. Sassi, B. Desiatov et al., "On-chip integrated, silicon-graphene plasmonic Schottky photodetector with high responsivity and avalanche photogain,” Nano letters, 16(5), 3005-3013 (2016). 